\title{
The Ethics of Biobanking: Key Issues and Controversies
}

\author{
Heather Widdows $\cdot$ Sean Cordell
}

Published online: 22 July 2011

(C) Springer Science+Business Media, LLC 2011

\section{Introduction}

The ethics of biobanking is one of the most controversial issues in current bioethics and public health debates. For some, biobanks offer the possibility of unprecedented advances which will revolutionise research and improve the health of future generations. For others they are worrying repositories of personal information and tissue which will be used without sufficient respect for those from whom they came. Wherever one stands on this spectrum, from an ethics perspective biobanks are revolutionary. Traditional ethical safeguards of informed consent and confidentiality, for example, simply don't work for the governance of biobanks and as a result new ethical structures are required. Thus it is not too great a claim to say that biobanks require a rethinking of our ethical assumptions and frameworks which we have applied generally to other issues in ethics. This special issue is dedicated to addressing these issues from the different perspectives of law, philosophy, medical ethics and sociology. This paper begins with a broad introduction to the "ethics of biobanking' which maps the key challenges and controversies of biobanking ethics; it considers; informed consent (its problems in biobanking and possibilities of participants' withdrawal), broad consent, the problems of confidentiality, ownership, property and comercialisation issues, feedback to participants and the ethics of re-contact.

The papers in this special issue were all presented at the eighth international workshop of the Tiss.EU Project on the theme 'Biobanking: Ethics, Governance and Regulation', at the University of Birmingham, UK, in June 2010.

H. Widdows $(\bowtie) \cdot S$. Cordell

Birmingham, UK

e-mail: h.widdows@bham.ac.uk 


\section{The Inappropriateness of Informed Consent for Biobanking}

In recent decades the appropriateness of informed consent as the 'gold standard' to ensure ethical treatment has been questioned both in therapeutic practice and medical research $[11,17]$. This is particularly the case when genetic information is at stake and in large-scale population studies-thus whether and what consent is appropriate in biobanking has been at the fore of this debate [4].

Informed consent has been the gold standard of informed consent since its introduction in the Nuremberg Code which requires that the research subject should know "the nature, duration, and purpose of the experiment; the method and means by which it is to be conducted; all inconveniences and hazards reasonable to be expected; and the effects upon his health or person which may possibly come from his participation in the experiment" [16]. These requirements were supplemented and clarified in the many versions of the Declaration of Helsinki. The latest of these, 2008 , adds that research subjects should also know the sources of funding, possible conflicts of interest, the intended benefits, any other relevant aspects and should have a right to withdraw.

Attaining these standards of informed consent is problematic for a number of reasons in biobanking. First, and an issue which applies to all genetic material and information, informed consent concerns only the individual and does not take account of other connected individuals; second, biobanking is future-orientated, thus consent cannot be 'informed' at the time it is attained as the nature of the future research is not yet known; third, biobanks are not $a$ research project, but rather a resource for many many research projects, thus making consent for each individual research project impractical to the point of impossibility; and fourth, it is not clear that the 'right to withdraw' can be fully respected in biobanking.

\section{Informed Consent Ignores the Rights and Interests of Connected Individuals}

A first reason for the inappropriate nature of informed consent as the ethical tool to ensure ethical practice in biobanking applies to all research using genetic material and information; namely, that informed consent does not take into consideration the risks and benefits of current or future research posed to third parties. Unlike other medical information, genetic information provides information about others in addition to the individual from whom samples are taken. For instance, genetic tests on one family member reveal information about the genetic status of other family members. For example, if an individual tests positive for a genetic condition, such as Huntington's disease, or as a carrier of the BRCA1 or BRCA2 (indicators for breast cancer) this information has relevance for family members (consanguineous relations may wish to be tested themselves, and sexual partners may desire the information when making reproductive decisions) [21].

Accordingly, who has access to genetic information and material is not only of concern to the individual from whom samples are taken as, "disclosure of genetic information by individual DNA donors also exposes information about others with similar genetic profiles" ([15], p. 376). The "key feature about genetic information is that it is typically information about a family, or even... about a larger community 
not just about an individual patient" ([3], p. 34). This is no less true in biobanking than in other areas of genetic research for, as Rothstein states, the risks of biobank research, "go beyond the individual human subjects to population groups with which the subject is associated as well as the general public" ([18], p. 90). Such risks include the discovery of information that will affect the health of the subject's genetic relations and the possible discriminatory use of the information discovered against certain cultural, geographical and age-related groups [1]. Thus ethical mechanisms are required which consider the rights and interests of those connected to the individual from which samples are taken, whether the family group or the public in general, as well as from the individual [20, 22, 23]. Informed consent clearly cannot take third party rights and interests into account and thus is not fit for purpose when it comes to the ethics and governance of biobanks.

The Future-Orientated Nature of Biobanks Precludes the Possibility of Being 'Fully Informed'

The second reason why consent for biobanking cannot be called 'informed' is quite simply because at the point of consent the information necessary to make consent 'fully informed' is not available. Thus the ethical worry is that if consent is to something as yet unknown, how can it be 'informed' so as to respect individual autonomy [12]? At the time of consent it is likely that there is little knowledge about what specific research will be done using the research subject's data. Thus, it is impossible for the research subject to be informed about the purpose, methodology, risks, funding sources and all the other requirements of informed consent. The Declaration of Helsinki, and other models of informed consent, assume that consent takes place at the beginning of a specific research project and the aims, benefits and risks are fully known. This is simply not the case in biobanking, at best only general areas of research are known. Sometimes these areas will be more focused, for instance, a biobank set-up using donations from a patient community to research a particular disease is likely to be able to anticipate future research projects with some accuracy, whereas a biobank like UK biobank, which is set-up to "build a major resource that can support a diverse range of research intended to improve the prevention, diagnosis, and treatment of illness and the promotion of health throughout society" ([19], p. 3), could not expect to predict future research with any degree of certainty. In such cases if there is not full information then there can never be fully informed consent. Accordingly, "because it is impossible for the donor to make an informed choice about the risks and benefits of unspecified future research protocols, such permission should never be called informed consent" ([25], p. 1180). Thus, the future-orientated nature of biobank research makes it is impossible for consent in biobanking ever to be informed consent.

\section{Biobanks are Not a Research Resource Not a Research Project}

The third problem is that biobanks are not a research project but rather a 'resource for research' or 'a research library'. Therefore, one solution to the problems of attaining consent for future projects is to ask for informed consent not for 
participating in the biobank in general, but for the specific research projects. Thus rather than seek consent for biobank participation, consent is sought at the beginning of every specific research project which uses the biobank.

However, while this would meet the requirements of the Declaration of Helsinki to seek consent for each individual research project impractical to the point of impossibility, the sheer numbers make this unrealistic ([22], 85). Such a requirement would demand returning to the donors for every new study-and potentially for every subsequent study which drew on previous data. To do this would not only be administratively cumbersome but more importantly overly burdensome on the donors to the point of impossibility. Especially for biobanks which are large-scale and intended to build and expand over a significant amount of time, such a model of seeking consent at the beginning of every individual research project is just not possible. Accordingly biobanks have largely rejected informed consent and moved to other models of consent, such as broad and blanket consent, often supplemented with additional ethics and governance mechanisms.

\section{Problems of Meeting the 'Right to Withdraw' Criteria}

The final issue which makes informed consent problematic in biobanking is the requirement of the right to withdraw. The Declaration of Helsinki states that the research subject "must be informed of the right to refuse to participate at any time without reprisal" [6]. Depending on how participation is interpreted it is not clear that this is possible in biobanking projects, particularly those which are largescale. For instance, UK Biobank grants participants the 'right to withdraw': "at any time and without having to explain why and without penalty" ([19], p. 6). However, there are different possible understandings and levels of withdrawal. For instance UK biobank offers three withdrawal options: 'no further contact', 'no further access' and 'no further use'. However even the most extreme formal withdrawal ('no further use') is qualified: "UK Biobank would destroy their samples (although it may not be possible to trace and destroy all distributed anonymised sample remnants) and would only hold their information for archival audit purposes" ([19], p. 9). In addition, although this most complete withdrawal would prevent information being used in further analyses "the participant's signed consent and withdrawal would be kept as a record of their wishes" and it may of course "not be possible to remove their data from analyses that had already been done" ([19], p. 9). It is also not possible to stop data being used in subsequent studies which build on the results of previous research done based on biobank samples and information. Given the nature of biobanks, what is possible is limited. However, participants may not regard 'no further use' as full withdrawal; they might imagine full withdrawal would allow them to remove all records of their involvement. Thus, while the greatest possible withdrawal options are available it is questionable that biobanking meets the withdrawal requirements of informed consent as set out in the Declaration of Helsinki.

Taken together these arguments show why biobanks, particularly large-scale and long-term biobanks, cannot meet the standards of informed consent as set out in the Declaration of Helsinki. In addition, especially given the ethical concerns about the 
effectiveness of informed consent for protecting all persons (including third parties), rejecting informed consent and seeking other ethical frameworks has become a key-indeed probably the key_ethical issue of biobanking.

\section{Broad Consent}

There are a number of possible ethical frameworks which could be adopted in preference to informed consent. The one most discussed in the literature is broad consent. Some ethicists do argue that broad or blanket consent alone is a solution to the problems of informed consent in biobanking, but most do not see this as an ethical solution on its own as it offers so little protection and few guarantees. In effect broad consent can be regarded as permission to do anything the recipient biobank sees fit with genetic material. Whilst it has been suggested that broad consent 'can be seen as a means of maximising autonomy' ([13], p. 196) in the biobanking context, it could be taken to be its opposite: a wholesale abrogation of individual autonomy, particularly with regard to knowledge future use of their material. The worry here is that in giving broad consent, the donor is being informed of little more than the fact that they have relinquished the access to knowledge about its future usage. Actual models of broad consent have sought to assuage this concern; for example the UK Human Tissue Act (2004) maintains the right of the donor to withdraw their sample at any time, and recommends that they be updated with any significant change of the purpose to which material may be put, for example 'if their samples will or could be used for research involving the commercial sector'. (2004 para 80) However, given the unknown nature of future biobank research this alone is rarely thought satisfactory. In addition to this weakness broad consent, like informed consent, is given by the individual and thus similarly fails to take into account the rights and interests of third parties. That is, broad consent, like informed consent, is sought only from the individual from whom samples are taken and not from those connected to them. Thus what has been generally suggested is that broad consent is supplemented by other ethics and governance mechanisms which ensure that ethics is ongoing over time and that the rights and interests of wider groups, beyond just the participants, are respected [2, $12,24]$.

UK Biobank is an example of such a model. Broad consent is used and is supplemented by other ethics and governance mechanisms which are ongoing for the lifetime of the project. Thus there are safeguards for both the participant (and over time) and for those who do not participate but who have a wider interest in the biobank: The participant is protected by both the broad consent which requires that UK Biobank must conform with the terms of the original consent and by the additional ethics and governance mechanisms which ensure that this continues over time; and connected persons and third parties are protected by the additional ethics and governance mechanisms which ensure that research happens in the public interest. In the words of the ethics and governance framework "UK Biobank will serve as the steward of the resource, maintaining and building it for the public good in accordance with its purpose" ([19], p. 12). 
Thus, UK Biobank's participants give broad consent that their samples can be used in ways which fit the stated purpose of UK Biobank; that is, to "build a major resource that can support a diverse range of research intended to improve the prevention, diagnosis, and treatment of illness and the promotion of health throughout society" ([19], p. 3). When asked to consent participants are asked on the basis that participation is "an opportunity to contribute to a resource that may, in the long-term, help enhance other people's health" ([19], p. 5). While this is a broad statement of its aims, it does not permit any research at all to be carried out on the participants samples (the worry that broad consent is overly permissive). Research must fall within UK Biobank's stated aim-that "the resource is being used in the public interest" ([19], p. 13) scientific interest alone is not enough-and ongoing ethics and governance mechanisms are in place to ensure that this is the case.

Given the reliance on additional ethics and governance mechanisms, much depends upon the success of these and on how effective they are in holding UK Biobank to its promise to ensure that research is in the public interest. Determining the public interest in detail is potentially problematic, however, broad principles are not difficult to determine. For instance, research which posed a public health risk would be unacceptable-whatever the expected scientific benefit-as perhaps would be research leading to developments which the donor group would not have access to (for instance, due to a high cost). Thus the public interest must be a determining criterion when it comes to allowing access to the resource. In UK biobank this is required in the Ethics and Governance Framework which sets out the aims of UK Biobank, with which UK Biobank is bound to comply, and conformance with this is monitored by the Ethics and Governance Council (EGC). Thus the ethical focus is not only at the beginning of the research (at the point of consent) but, by incorporating additional ethical and governance mechanisms, occurs throughout the lifetime of the project or the research. The EGC's remit is:
"acting as an independent guardian of the Ethics and Governance Framework and advising the Board on its revision; monitoring and reporting publicly on the conformity of the UK Biobank project within this Framework; and advising more generally on the interests of participants and the general public in relation to UK Biobank" ([19], p. 15).

Thus, "the Ethics and Governance Council will keep use of the resource under review in order to advise on conformance with this Framework...to assure itself, and others, that the resource is being used in the public interest" ([19], p. 13). Therefore in this model broad consent is supplemented by the EGF and EGC, and the processes which UK Biobank adopts, such as the access policy, have to be in conformity with the EGF. Thus although 'broad' this consent is not empty-it is bound overtime, thus respecting both the participants and their consent, and it is has to be in public interest, thus going some way to respecting the needs of all, not only those of the participants.

In addition to the rethinking of consent which biobanks have necessitated there are a number of additional new ethical issues which biobanks raise; some of which are connected to the consent issues. There is not enough time to do much more than 
to list these and outline the ethical debate, but this in itself is sufficient to show why biobanking challenges traditional ethical assumptions and has been given so much attention in the field of ethics.

\section{Confidentiality}

A key feature of genetic information is that it is always potentially identifying, both of the individual who donates the sample, and of those related to them (both now and in the future). Thus, while identification can be discouraged-by various process of anonymisation-it can never be fully guaranteed as identification is potentially possible, for instance if samples are compared to a database. Thus, quite simply confidentiality cannot be fully guaranteed-no matter how anonymised the data is - as there is always a risk of identification. This risk grows as the number of databases grows and thus the possibility of identification increases [21, 22]. Thus while every effort can be made to annonymise date and to protect privacy in largescale biobanks which link to databases unintentional identification of individuals is always possible. Given this full confidentiality should never be promised in biobanking (or indeed in other areas of genetic research, although the linkages and scale of biobanking make the risks more likely). This is of course in tension with the traditions of medical ethics which, since the time of the Hippocratic Oath has promised confidentiality.

\section{Property and Profit}

A second additional issue is about property and profit. The Ethics and Governance Framework of UK Biobank states clearly (and three times) that participants have no property in their samples. The view that participants have 'no property' in their genetic samples has been based on a traditional understanding that body parts, once detached, are res nullius, 'no one's thing'. Interestingly this traditional understanding has more recently been challenged by advances in technology, particularly where bodily materials are stored expressly for future usage of the donor in ways that was once not viable $[8,26]$.

However, there are reasons for adhering to the 'no property' rule when it is applied to samples in biobanks: Some reasons are practical as allowing property in biobank samples would hinder the practice of research to the extent it would be likely to become untenable; and some are ethical, they reject property in samples as this might lead to the commodification of persons [5]. Yet while there are good reasons for this view there are some dangers attached, particularly with regard to the potential for commercial companies to make profits (and potentially very large profits) from the research done on altruistically donated samples. Such profit making is likely to feel to participants to be at odds with the public good rhetoric regarding the aims of biobanks.

Some biobanks have responded by trying to show the compatibility between public altruism and private profit. For instance, UK Biobank states that "The 
biotechnology and pharmaceutical industries can play an important role in realising health benefits in a practical sense by developing and improving the use of biomedical products. Commercial companies and other research endeavours that stand to make a profit will, therefore, be allowed access to UK Biobank if their proposal falls within the UK Biobank purpose and complies with the usual scientific and ethics requirements" ([19], p. 18). In addition, biobanks (including UK Biobank) are considering profit sharing methods or at least some mechanism for ensuring that any profits gained does not turn into excessive profiteering. Others make a distinction between different types of users; for instance, between commercial and non-commercial users. Thus CARTaGENE operates a "differential cost scheme for public, private and international researchers” ([7], p. 112).

The success of these various approaches in terms of satisfying participants and the public and in terms of reducing and prevent excessive profiteering is yet to be seen. However, getting this right is crucial for the survival of biobanking as participants in biobanks have a tendency to regard their contribution as a duty to other and part of being a good citizen. For instance in an Australian study Allen and McNamara found that "consent to participate in biobank research is framed as the moral act of a responsible citizen, which reinforces self identity and in the process entrenches the perception of research as a public good" [1]. Thus this trust is maintained by the ethics and governance procedures which accompany informed consent, but also by ensuring that the public good is actually served. It is doubtful that participants will view undue profiteering by private companies as serving the public good and this may lead to mass withdrawal (the main threat to the success of a biobanking project). How to address worries about unreasonable profiteering remains an issue for biobanks and the jury is out on which is the best method and it is to early to tell which approach will end up as standard.

\section{Feedback}

Another ethical issue which arises in research in general, but which is exacerbated in biobanking, again because of the numbers involved, is that of whether or not to feedback to participants 'incidental findings'. While there is no standard definition 'incidental findings' they can broadly be described as "observations of potential clinical significance unexpectedly discovered in healthy subjects, or in patients, recruited to brain imaging research studies and unrelated to the purpose and variables of the study" ([9] p. 783). The key issue is that they are 'incidental'ascertaining information of clinical significance is not the aim of biobanks, rather collecting material for later research is.

There are two points at which the feedback of incidental findings might be an issue: when material is collected (if this is done specifically for the purposes of use in the biobank) and at some future point in research. For instance UK Biobank conducts its own collection of material which includes the taking of physical samples, asking lifestyle questions, and then linking this information to healthrelevant records. UK Biobank's policy to date has been to offer minimal feedback. Participants are informed that the collection of material is not a clinical 'health 
check', but rather collection for the purposes of research, and thus they should not expect feedback. However, even in this minimal feedback model it is judged that "even in this research context, there may be occasions when staff consider there to be a professional or ethical obligation to draw attention to abnormal measurements (such as elevated blood pressure) or incidental findings (such as possible melanoma)" ([19], p. 7). Thus while rare there are instances where it will be suggested to participants by UK biobank staff that they should contact their GP or other relevant health professional. Incidental findings can also arise in future research; and the likelihood that results are attained which have relevance for an identifiable individual becomes more likely as links to additional databases increase, so increasing the likelihood of unintentional identification. The rationale given for the low level of feedback relates both the quality of the feedback, its usefulness and its possible negative effects ([19], p. 7).

However, the failure to feedback has been criticised and the ethical justification of limited feedback is being increasingly questioned. For instance, Johnston and Kaye have argued that there is a duty to feedback to individual UK Biobank participants for both legal and moral reasons. They argue that this is particularly the case in the "rare situation ... where the research reveals that an individual or his family is at risk of a serious yet treatable condition" ([10], p. 267). The pressure to feedback and the ethical questioning of the claim that feedback is only rarely necessary at best is likely to increase as research progresses and as additional enhancements are added to biobank resources. For instance, UK biobank is in the process of attaining funding to carry out Magnetic Resonance Imaging (MRI) on a sub-section of the original participants to enhance the biobank resource. It is currently an open question whether the no (or very little) feedback policy will continue if this is permitted. Unlike the initial visit the data which will be collected from the MRI is not easily available elsewhere (as blood pressure results are, for example), thus this scan might be the only point at which access to these results might be possible. Also, as an MRI scan is generally regarded as a diagnostic tool and used reveal any significant health problems, there is likely to be increasing expectations on the part of the participants that they will be told if anything is wrong (already a problem even at the base line assessment) [14]. Given these trends it is likely that feedback will become an increasingly important ethical and policy debate in the governance of biobanks.

\section{Ensuring Participation, Representation and the Maintaining of Trust}

As discussed, the biggest threat to biobanks is the mass withdrawal of participantsif participants withdraw the biobank fails and if this happens early in the process then the substantial set-up costs will be lost and there will be no gain at alltherefore ensuring that participants and the wider public are appropriately engaged with biobanks and that trust between these groups is maintained is crucial. However, there are different models of possible engagement, from those where participants (and in some biobanks the public) are very involved in the biobank-in terms of directing future research and enhancements to the resource-to a model where there 
is no engagement as such but the biobank merely keeps participants informed. These range from participant and/or consultative panels, patient and public decision-making groups, citizens inquiries, participant and public shareholding in the biobank, to participant and public seats on the biobank board. Just as with property and profiteering there is as yet no one way which is viewed as best practice and, as in so much of biobanking practice, what is good ethics and governance is yet to be determined in this area.

\section{Re-Contact}

Like feedback, recontact is becoming an increasingly important issue. There is little yet written on how recontact should be managed. But like issues of participation and consent there is clearly a balance to be found between ensuring that participants are as informed and included as they expect without over-burdening them. Just like biobank samples, the ability to 're-contact' should be seen as a 'finite and depleteable resource'. Thus, re-contact should be limited and mechanisms need to be devised to ensure that this is still possible in the long-term. The likelihood is that this will require biobanks to prioritise between research projects, however, exactly how this will be done is another area in which there is currently no standard procedures for biobanks to adopt.

\section{This Issue}

We have said that informed consent, problems it faces in biobanking and the need for alternative models are a key source of issues in biobanking ethics. Unsurprisingly then, several of the papers in this issue concern or touch on the questions of informed consent. In their analysis of the 'New Belgian Law on Biobanks' Sigrid Sterckx and Kristof Van Assche note some of the legal and ethical implications of this law regarding consent, for example that it allows for 'presumed consent' to research in the case of 'residual materials'. Interestingly, and worryingly for Sterckx and Van Assche, such material is deemed 'residual' if it was extracted for diagnostic purposes, but unless the patient explicitly requests otherwise their consent to its use in research is, apparently, not merely 'tacitly assumed' but 'presumed'.Tacit consent to certain instances of research is one thing when a donor has agreed that their material at least may be used in some kind of research, but it is quite another in cases where no such agreement obtains.

In 'Respect for Autonomy: its Demands and Limits in Biobanking', Iain Law takes up the question of autonomy, more specifically the notion of autonomy employed in discussions of informed consent and the level of information required to respect such autonomy. If we are to make respect for donor autonomy a cornerstone of biobanking ethics, then we need to be clear about what it is we mean by autonomy and what a requirement to respect it does and does not entail. The constraints of autonomy in the context of biobank research, on Law's view, entail only that a consenting person is not deceived, so will not entail a requirement 
actively to supply full information at every stage of a biobank's activity involving participants, for example. An interesting implication of his paper is that talk about autonomy is often loose, and there is no reason to think that it is any more careful in bioethics. Hence the issue of informed consent in relation to biobank research is one where it should be more precise.

Søren Holm looks specifically at the question of participant withdrawal from biobanks. He begins his paper 'Withdrawing from Research-A Rethink in the Context of Research Riobanks' by observing that whilst it would be impossible to account for everything that has been written on the conditions for participation in research, it might prove similarly difficult to find literature on the conditions for withdrawal. Having granted that there are some good historical reasons and principles to defend the 'established view' of the right to withdrawal, on which that right is: absolute; unconditional; immediate; and complete, he argues that there may be cases in which components of this right should be relaxed, so on this view the right to withdraw is not inalienable, nor is it necessarily unconditional.

Other papers in this issue start or overlap with questions of consent, whilst also considering some of the other problems outlined above (1-7). Sean Cordell motivates his account of 'The biobank as an Ethical Subject' by taking the debate over consent as a crystallizing example of the broad ethical and socio-political terms in which biobanking issues are conducted, where for example individual rights are balanced with public health and future benefits to entire populations. What is missing from this debate, he then claims, is a more determinate understanding of the biobanking institution as an entity with certain internal standards of conduct derived from 'what it is there for', from its being 'fit for purpose'. When that purpose is construed as essentially connected to certain human goods of health and research in ways specific to biobanking, it can be understood as something which places certain ethical limits on its activities.

Continuing with how a biobank is understood or perceived, an important question is that of how public participants view their own relationships with a biobank. This question relates most conspicuously to the topics of feedback (5), trust and continuing participation (6) and the extent to which participants should, or might expect to be re-contacted (7). Mairi Levitt focuses on these topics in 'Relating to Participants: How Close do Biobanks and Donors Really Want to Be?' She looks at the ways in which biobanks appeal to donors and present the co-operative enterprise of the biobank and its projects, and at how participants themselves respond and envisage this relationship. An interesting conclusion is that for a biobank getting the 'right' relationship could mean more than only establishing the one that, for example, ensures continued participation. This is because different kinds of relationship bring with them different expectations and obligations. So whilst public engagement is crucial, so is considering the terms of that engagement-for example are we 'acquaintances' or more like 'friends'? - and being clear about what kind of feedback and degree of confidentiality is to be expected.

All of the ethical issues raised here should of course be those considered in constructing and implementing actual ethical guidelines and regulations. An important and overarching issue in this respect is about who does the constructing and implementing and on what basis. This is tackled by Jean McHale in 
Accountability, Governance and Biobanks: The Ethics and Governance Committee as Guardian or as Toothless Tiger'. In the context of the UK, McHale explores whether it is correct to regard Ethics Committees as such "toothless tigers", and considers alternative structures which may provide a more appropriate regulatory paradigm for the future. More specifically the question of whether the ethics committee serves as 'legitimator' of research or as a means of ensuring accountability (or both). Acknowledging good reasons of objectivity and independent perspectives for ethics committees to exist, McHale expresses the worry that the ethics committee is nevertheless all too 'toothless'. Hence her article provides a critical view of the legal framework within which ethical issues and recommendations are discussed, and of how this framework might be improved.

As can be seen just from this overview, the topic of biobanking presents significant and distinct ethical challenges. As stated in the introduction, the aim of this special issue is to advance the important and developing debates about these challenges in a number of ways. In addition it is also hoped that the wide and interdisciplinary nature of this collection will underline and reflect both the interest in, and the importance of, biobanking, a topic that potentially impinges on so many areas of human life.

\section{References}

1. Allen, J., \& McNamara, B. (2011). Reconsidering the value of consent in biobank research. Bioethics, 25(3), 155-166.

2. Austin, L. M., Lemmens, T. (2009). Privacy, consent, and governance. In K. Dierickx \& P. Pascal Borry (Eds.). New challenges for biobanks: Ethics, law and governance, 2009. Available at: http://ssrn.com/abstract=1538512.

3. Brock, D. W. (2001). Genetics and confidentiality. American Journal of Bioethics, 1(3), 34-35.

4. Casado Da Rocha, A., \& Seoane, J. A. (2008). Alternative consent models for Biobanks: The new Spanish law on biomedical research. Bioethics, 22(8), 440-447.

5. Cordell, S., Bellivier, F., Widdows, H., Noivelle, C. (2011). Lost property? Legal compensation for destroyed sperm: A reflection and comparison drawing on UK and French perspectives. Forthcoming in Journal of Medical Ethics. (Published Online June 13 2011. 10.1136/jme.2010.042036).

6. Declaration of Helsinki (2008). Section 24. http://www.wma.net/en/30publications/10policies/b3/ index.html.

7. Fortin, S., Pathmasiri, S., Grintuch, R., \& Deschenes, M. (2011). Access arrangements' for Biobanks: A fine line between facilitating and hindering collaboration. Public Health Genomics, 14, 104-114.

8. Harmon, S. H. E., \& Laurie, G. T. (2010). Yearworth Vs North Bristol NHS Trust: Property, Principles, Precedents and Paradigms. Cambridge Law Journal, 69, 476-493.

9. Illes, J., Kirschen, M. P., Edwards, E., Stanford, L. R., Bandettini, P., Cho, M. K., et al. (2006). Ethics. Incidental findings in brain imaging research. Science, 311, 783-784.

10. Johnston, C., \& Kaye, J. (2004). Does the UK Biobank have a legal obligation to feedback individual findings to participants? Medical Law Review, 12, 239-267.

11. Manson, N. C., \& O’Neill, O. (2007). Rethinking informed consent in bioethics. Cambridge: Cambridge University Press.

12. Maschke, K. J. (2006). Alternative consent approaches for biobank research. Lancet Oncology, 7 , 193-194.

13. McHale, J. (2006). 'Appropriate Consent' and the use of human material for research purposes: The competent adult. Clinical Ethics, 1, 195-199.

14. Miller, F. G., Mello, M. M., \& Joffe, S. (2008). Incidental findings in human subjects research: what do investigators owe research participants? Journal of Law and Medical.Ethics, 36, 271-279. 
15. Mitchell, G. R., \& Happe, K. (2001). Informed consent after the human genome project. Rhetoric and Public Affairs, 4(3), 375-406.

16. Nuremberg Code. (1949). Directives for human experimentation. http://ohsr.od.nih.gov/guidelines/ nuremberg.html. 2008.

17. O'Neill, O. (2002). Autonomy and trust in bioethics. Cambridge: Cambridge Univerity Press.

18. Rothstein, M. A. (2005). Expanding the ethical analysis of biobanks. Journal of Law, Medicine and Ethics, 33, 89-101.

19. UK Biobank Ethics and Governance Framework. (2007). http://www.ukbiobank.ac.uk/docs/2007 1011_EGF_Version_3_1_0October_2007withTOR.pdf.

20. Widdows, H. (2007). Conceptualising the self in the genetic Era. Health Care Analysis, 15, 5-12.

21. Widdows, H. (2007). Reconceptualising genetics: Challenges to traditional medical ethics. In C. Lenk, N. Hoppe, \& R. Andorno (Eds.), Ethics and law of intellectual property: Current problems in politics, science and technology. Aldershot: Ashgate.

22. Widdows, H. (2009). Constructing communal models of governance. In H. Widdows \& C. Mullen (Eds.), The governance of genetic information: Who decides?. Cambridge: Cambridge University Press.

23. Widdows, H. (2009). Between the individual and the community: The impact of genetics on ethical models. New Genetics and Society, 28(2), 173-188.

24. Winickoff, D. E. (2003). Governing population genomics. Jurimetrics, 43, 187-228.

25. Winickoff, D. E., \& Winickoff, R. N. (2003). The charitable trust as a model for genomic biobanks. New England Journal of Medicine, 349, 1180-1184.

26. Yearworth \& ors v Bristol NHS Trust (2009). EWCA Civ 37 (04 February 2009). 